\title{
SAGA Reloaded: Towards a Generic Platform for Developing Cooperative Applications
}

\author{
Benjamim Fonseca ${ }^{1}$, Hugo Paredes ${ }^{2}$, João Paulo Sousa $^{3}$, F. Mário Martins ${ }^{4}$, Eurico Carrapatoso ${ }^{5}$ \\ ${ }^{1}$ CITAB/Universidade de Trás-os-Montes e Alto Douro, Quinta dos Prados, Vila Real, Portugal \\ ${ }^{2}$ Universidade de Trás-os-Montes e Alto Douro, Quinta dos Prados, Vila Real, Portugal \\ ${ }^{3}$ ESTGM-IPB, Departamento de Informática e Comunicações, Mirandela, Portugal \\ ${ }^{4}$ Universidade do Minho, Departamento de Informática, Campus de Gualtar, Braga, Portugal \\ ${ }^{5}$ Universidade do Porto, Faculdade de Engenharia, Porto, Portugal
}

\begin{abstract}
Groupware specification and development has always been a complex task, requiring special attention to issues such as notification of cooperative actions and ensuring consistency of shared data. Some years ago SAGA was developed as a framework to build groupware applications based on a set of core web services that provide the most common cooperative functionalities. Despite its potential, the last few years brought some technological developments that placed new challenges. This paper presents a new generation of the SAGA platform that adds to the original framework features that emerged recently, namely the regulation of social interaction, incorporation of new communication technologies, connectors to several external services and interaction environments, and the addition of contextual information.
\end{abstract}

Keywords: Groupware, Collaborative Platform, SAGA.

\section{Introduction}

Computer Supported Cooperative Work (CSCW) is a topic that has been studied in the last decades and a myriad of scientific publications have been produced concerning issues ranging from theoretical aspects, like classification of applications or sociological studies, to implementations of cooperative tools or frameworks.

The implementations of cooperative applications, usually referred to as groupware, require special attention to multiple issues. First, it is necessary to carefully evaluate the real need and opportunity for such tools. There are groupware applications that were never used because their potential was over-evaluated - there is no need at all or some other tool or combination of commonly used tools can do efficiently the job. It is also possible to find several groupware tools that were not designed accurately in terms of functionality, usability or technological support, and thus failed to succeed, despite the correct evaluation of its capabilities and opportunity [13]. Moreover, it is also common to find resistance of users and organizations to adapt to new technologies that somehow change their work routines, even if they can benefit from this change.

Among the technological aspects to consider when developing cooperative applications, the following deserve special attention: communication among participants, sharing of information, notification of cooperative actions, authentication, concurrency control and user's awareness. With these issues in mind, several frameworks have been developed to enable the construction of groupware applications. One of these frameworks, SAGA (web Services Architecture for Groupware Applications) [11] was built using web services with the aim of providing a set of core cooperative services that can be combined to develop virtually any cooperative application.

SAGA revealed an interesting potential, but it now faces new challenges. For example, SAGA was targeted to client-server applications with a focus on the development of synchronous groupware, despite its ability to also deal with asynchronous cooperation. However, in the last years, peer-to-peer solutions are gaining an increasing importance.

In several situations involving social interaction or a specific flow of activities, regulation mechanisms [31] are required to avoid confusion, incorrect use of tools or drifting of tasks' execution. Cooperative applications require generally a high degree of social interaction, so the availability of regulation mechanisms can be important to build robust groupware.

Mobile computation has an increasing community of users and is a natural field for cooperative applications. However, some mobile devices have important resources' limitations, so it is desirable that most applications can be used dynamically whenever available in the form of services. These services, if correctly specified, have the potential of being combined dynamically to produce richer services. In this scenario it is also interesting to have the possibility to compose services dynamically depending on the current user's context (location and hardware, for example) [35]. To achieve this it is also important to add metadata to the description of services and shared information. 
In this decade several social interaction applications have emerged that transformed the web into what we usually call Web 2.0. Among these applications, virtual worlds have been growing fast in the last few years, namely Second Life, which already has a vast community of millions of users. The 3D environment provided by virtual worlds open new opportunities to empower synchronous cooperation, through its strong visual impact and immersive capabilities.

This paper presents an ongoing work of reengineering SAGA with solutions that were driven by the new challenges described herein. In the next section, the basic background issues are presented, giving an overview of the original framework and contextualizing the main motivations to introduce changes in SAGA. Then, the new architecture is presented, giving special attention to the new functionalities and associated technologies. The paper ends with some considerations on the changes introduced in SAGA and the developments further ahead.

\section{Background}

In the current organizational context of companies and institutions, one success factor is the ability to effectively realize teamwork. This fact has raised the interest of organizations in applications of Computer Supported Cooperative Work (CSCW). This kind of applications, usually referred as groupware, supports several group tasks, which can be divided in four main categories: jointly authored pages, streaming technologies, information access tools and aggregated systems. [24].

The development of groupware applications has several issues. Hua et al. consider five main issues in the design of CSCW systems: dynamic group forming, group awareness, group multicasting, QoS guarantee and session integration [5]. In fact one of the most important issues in groupware is the existence of an environment that is shared among team members. This environment may include documents, shared whiteboards and shared pointers, among others. Groupware applications must have mechanisms to distribute cooperative events produced by members in the shared environment. Usually, the shared environment coexists with the private environments of each member, imposing the availability of diverse management mechanisms to control information access. To ensure consistency of the data being shared, special care must be taken regarding concurrency control, implementing mechanisms like atomic transactions, locks, versioning, token passing or voting systems.

Usually, groupware tries to support teamwork in the most successful way, providing means for information sharing, for its joint manipulation and for communication between cooperating participants. However, the architecture of cooperative applications is based often on proprietary solutions, which do not address issues of flexibility, interoperability and support of legacy systems.

Groupware system can be divided into two main classes: centralized and distributed [2]. During the last decade, several frameworks have been developed to enable the construction of groupware applications. Examples of such frameworks are CBE [19] MetaWeb [36], Mushroom [18], Collaboration Bus [2], Habanero [4], Agilo [15], AORTA [29], DOORS [32], ANTS [21] and Artefact [3]. These frameworks are based on a centralized architecture and the major flaws of all them are the lack of interoperability support and the inability to integrate legacy applications. At the beginning of this decade, a new technology emerged, Web Services, promising a new degree of interoperability. Based on this technology, a new platform, SAGA, was specified and developed which included services that try to foster reusability and shorter development periods. Fig. 1 depicts the overall architecture of SAGA, showing the web services that constitute the core functionalities that can be used to build cooperative applications. The Authentication Service controls the access to the cooperative applications and services and the Users Directory enables cooperators to be aware of each other's activities. The Events Notification Service offers users the possibility of being notified of each other's actions during cooperative sessions. A critical issue of cooperative applications is the ability to guarantee consistency of the data being shared and the Concurrency Control Service contemplates several concurrency control policies for this purpose. All information to be shared is stored in the Group Storage module and accessible through the Information Repository. The Applications Repository gives access to the cooperative applications stored in the Group Storage, enabling on demand download and activation of the latest versions of the applications that are needed for the tasks currently assigned. Despite its positive reception from the groupware community, SAGA still lacks the

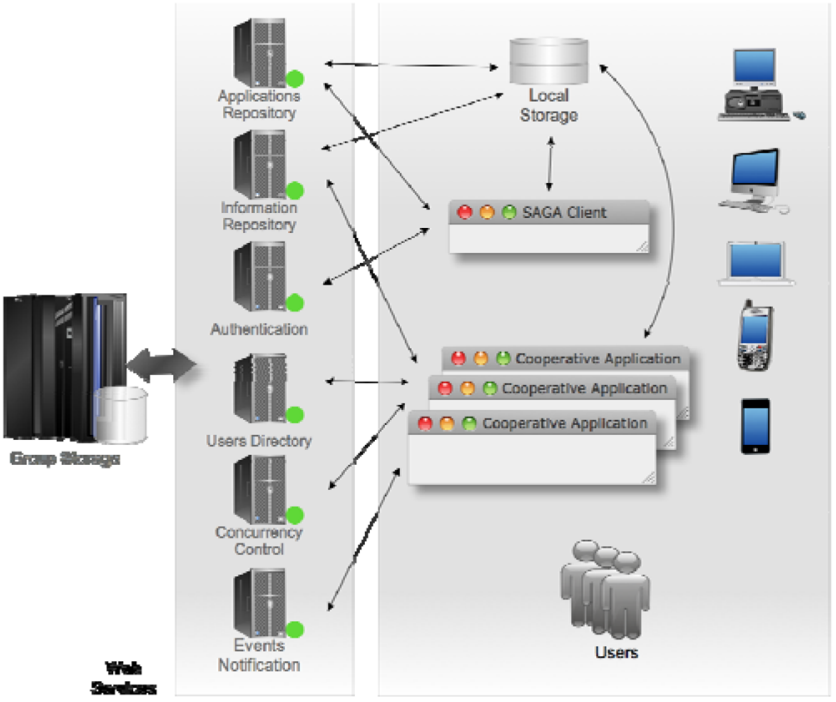

Fig. 1 - Original SAGA architecture 
inclusion of some issues that can make it a better solution.

Truly distributed frameworks have emerged in the late 90's with the publication of the Freenet protocol [6] and the development of peer-to-peer (P2P) applications which are characterized by their load balancing, robustness and self-organization. Distributing the core processing of the system, $\mathrm{P} 2 \mathrm{P}$ applications solve the server bottleneck problem that usually occurs in clientserver applications. In what concerns groupware applications, Bao-Qing et al. [2] argue that "collaboration work based on P2P solves the problem of Architecture Mismatch [7] between the traditional cooperation architecture and the cooperation view provided to users".

$\mathrm{P} 2 \mathrm{P}$ based groupware systems can be divided in pure $\mathrm{P} 2 \mathrm{P}$ systems, where every peer has the same capabilities and responsibilities in the system, and hybrid systems, that incorporate server elements in the system, facilitating the communication between peers [16]. Xia et al. also consider three types of the P2P model in cooperative systems: broker-mediated file sharing, peerto-peer file sharing and cycle sharing.

The diversity and complexity of work methodologies in organizations is increasing and the participants' responsibilities are not always statically defined. Hence, the execution of tasks by several persons, which may not always play the same role in the execution of a certain type of tasks, is relatively frequent. Furthermore, during the execution of a task, one person may want to consult others, regarding specific issues or to obtain approval from upper levels of the organization. This regulation and coordination problem is subject of study in several areas, namely Human Computer Interaction [20], Artificial Intelligence [9] and CSCW [10]. The Social Theatres (ST) model [31] was proposed as a basis for the development of regulated virtual social interaction environments, to better serve and solve the social interaction regulation and coordination problems. Generically, the concept of ST expands the theatrical interaction model to daily activities of social interaction in virtual interaction environments. The focus on ST aims to capitalize from its well known and easy to understand interaction model. There are roles, interaction flow and rules, which are the foundations of the proposed virtual interaction model. The interaction model is implemented by the ST supporting architecture that, among other features, regulates the virtual interaction, adapts to the user's interaction capabilities and technology and may also adapt the virtual interaction environment at runtime.

Associated to the knowledge of the behavior in the environment the team members can be assisted by the history of the actions performed by others or even by themselves in the past. In this context, an important part of a cooperative system is its collective memory. A groupware tool that can register the most important moments that occur during the team's interaction and infer knowledge in order to support the execution of future task would be a helpful assistance to team member in their tasks. Ackerman and Mandel [1] argue that "memory systems can provide additional and necessary support services for organization and communities" assisting and increasing the performance of important tasks, apart from memory that is used for the normal system runtime.

As mentioned before, group awareness is one important issue in collaborative applications. In order to work together, team members need information about the environment, namely who is present, actual activities and shared artifacts [12]. With the emergence of mobile environments, which enabled teams to cooperate while on the move, awareness information became a hot topic in the scientific community leading to further developments in context-aware computing. These systems have the ability to "adapt according to the location of use, the collection of nearby people, hosts, and accessible devices, as well as to changes of such things over time" [34]. The fusion of context aware applications and mobile CSCW gains particular interest in the situation where teams are geographically distributed and on the move [17], such as the exploration of dangerous areas and the management of emergencies [30]. In these environments, these combined applications are able to deliver contextualized information and service, context based automatic execution of services and the attaching of contextual information for later retrieval [8]. To achieve this, current $\mathrm{CSCW}$ infrastructures use reusable components and services that can be dynamically composed in order to adapt their functionalities according to users' and groups' needs [26]. However, to deliver service dynamic composition transparently to users based on contextual information, several issues have to be taken into consideration [35]. One of the most important issues in this problem concerns the classification and description of services, allowing composition mechanisms to check the composition syntactic and semantic integrity. Among others, semantic web services technologies [23] are one way to deliver the required metadata to services. Some frameworks and architectures such as SOCAM [14], MyCampus [33] and CACS [22] are examples of the usage of semantic information (ontologies) agents to discover, compose, select and execute automatically services based on context information.

It is evident that nowadays mobile applications are an important part to CSCW tools. However, more traditional approaches, as web applications, preserve some relevance in the area. The Web 2.0 concept [27], introduced a few years ago, contributed to an increasing interest in this kind of groupware tools. Web 2.0 defines a set of principles and practices that transforms the Web in a platform for collaboration and interaction, where everyone can participate. This environment is characterized by the contribution of its users, allowing the design of his "own Web", and consequently its 
expansion based on the supplied information in the form of users' discussions and opinions.

Moreover, an emergent field of groupware applications is related with its integration with virtual worlds. Research in collaborative virtual environments has been a hot topic for decades. The developments and success of virtual worlds such as Second Life [37] demonstrate that these environments have huge potential in new forms of human cooperation and interaction. Olivier and Pinkwart [28] point out the particular interest of virtual worlds supporting remote synchronous collaboration, awareness and social identity.

\section{Platform Specification}

The main goal of the original SAGA was to constitute a framework that would enable the development of cooperative applications through the composition of several core functionalities available through Web Services, providing a set of operations suitable to fit the requirements of every class of groupware applications. Current challenges in cooperative applications demand a new specification and adaptation of original requirements of the original SAGA framework. In this context we propose SAGA Reloaded (SAGA/R), a new framework sharing the main objective with the original framework, but having requirements adequate to current and foreseeing challenges.

The proposed platform inherits the traditional requirements of groupware applications from the SAGA framework, extending it to emerging human computer interaction (HCI) techniques and interfaces and challenging operating environments.

In what concerns HCI the requirements on the platform address:

- Interface technologies: including the integration and privileged interface with mobile applications, emerging collaborative virtual environments (CVE), such as Second Life or Active Worlds [38], Web 2.0 applications and other kinds of custom applications that are adapted to specific user requirements.

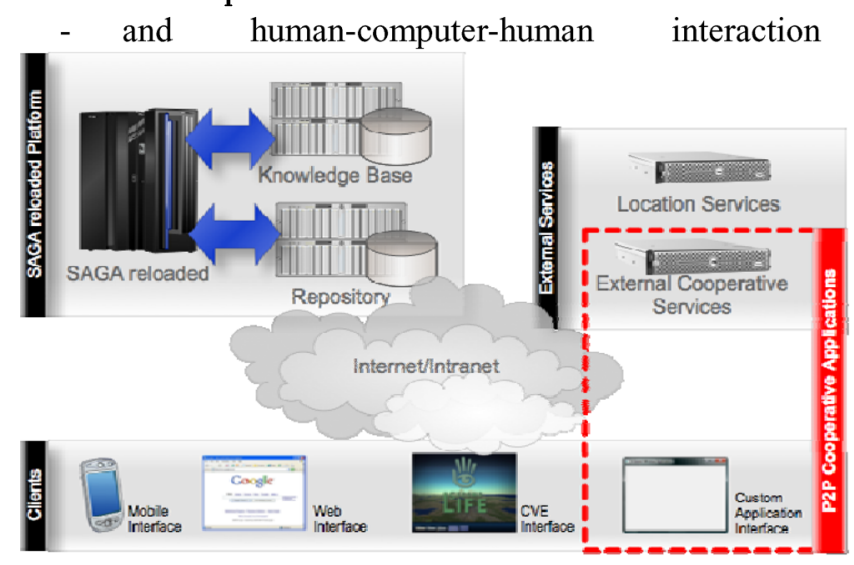

Fig. 2 - Physical platform architecture techniques: reflecting current strategies of interaction that include regulated and coordinated social interaction.

Challenging operating environments include the mobile environments, where occasionally connecting computing strategies are often used for on the move users, as well as asynchronous cooperation. Moreover, in this ubiquitous environments context aware applications are often a solution to some interaction and cooperation problems, addressing tools suitable for real time situations.

Based on these requirements, from the physical perspective the proposed platform is organized in three blocks (Fig. 2): clients, external services and SAGA/R platform; and a $\mathrm{P} 2 \mathrm{P}$ cooperative applications metablock.

The clients' block includes the possible clients for cooperative application hosted in the SAGA/R platform, such as interfaces for mobile, Web, CVE and custom applications. The external services block incorporates the services that are external to the platform and can be included in runtime using wrapper connectors. Examples of these services are third-party cooperative services and location services. A meta-block of P2P cooperative applications is defined aggregating the external cooperative services and the custom application interface, defining the application blocks that can be integrated in order to create hybrid P2P cooperative applications. Finally, the core of the architecture relies on the SAGA/R Platform block, where the platform itself and auxiliary services are located. The application core of the architecture is delegated in three servers: knowledge base, repository and SAGA/R. The knowledge base guarantees the management of knowledge for the regulation services of the platform, the repository stores all the groups' data needed for the cooperation applications and the $\mathrm{SAGA} / \mathrm{R}$ acts as innkeepers of the logical application layer of the platform.

From a logical perspective, the SAGA/R platform relies on four functional layers (Fig. 3), organized by their abstraction granularity: services layer, application

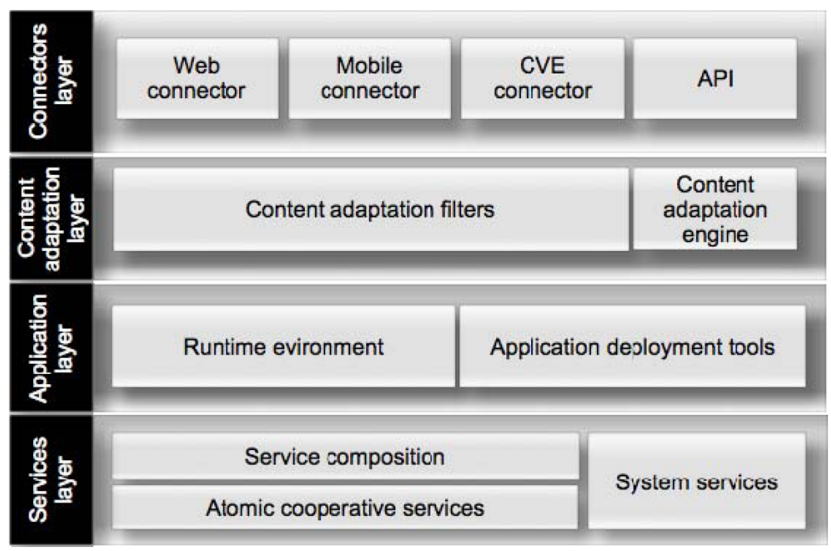

Fig. 3 - Layered model for the new SAGA platform 
layer, content adaption layer and connectors layer. The first two layers are concerned with the cooperative applications execution and their runtime; and the last two are oriented to the communication and integration of the platform with the environment.

The lowest layer (services layer) hosts the atomic components of the platform that confers the basic functionalities to the system: the services. Relying on a core of services, it allows the platform to be modularized in order to be adapted to each particular cooperative situation. The platform services were divided by their functionality and complexity. Moreover, there are a set of basic cooperative services, system services and a service composition component. Basic cooperative services include instant messaging, file sharing, group editor, workflow and task management, calendar, whiteboard, history and regulation services. Apart from this set of services other external services can be included using the connector service that is part of the system services. This service allows dynamic wrapping of external services to be included in the core services of the platform, as the connection to external location services. System services also include backend connectors in order to assure the connection management between the platform and the system repository knowledge base. Two of the most important services in the system, inherited from the original SAGA platform, which provide vital cooperative functionalities to the SAGA/R platform, are included in this block: the event notification service and the concurrency control service. Another particular service included in this set is the context management service which allows the creation of cooperation scenarios and their association to the core cooperation services. Contexts can be divided in global or specific contexts, depending on the visibility of services and data to other contexts. Other services that are part of system services are authentication, time, user directory and groups' management services.

System services and basic cooperative services can be composed in order to create complex cooperative services that will support the applications. This function is associated with the services composition component that, based on the services metadata, provides the composition of services and performs the required syntactic and semantic verification of the process. The platform defines a set of composed cooperative services for runtime support namely the awareness service and the brainstorming service. The awareness service supports context aware applications and is composed of the context, users' management, calendar and time services. Moreover, the brainstorming service is a common service included in cooperative applications and is composed of whiteboard, instant messaging, group editor and file sharing services.

Cooperative applications can be specified by the composition of basic cooperative services, system services and composed services. The applications are deployed on the application layer, using the application deployment tools component, responsible for the creation of the necessary runtime structures in the application runtime environment for the execution of the application. The application runtime component has as main function the management of the runtime structures of the cooperative application in their execution cycle.

The two upper levels assure the connection of the platform with its environment. The content adaptation layer is responsible for adapting the cooperation contents to the requirements of the client application as well as to the characteristics of the user interaction device, providing accessible contents according to the user profile. To achieve this functionality, the layer is composed of two components: a set of content adaptation filters, that adapt the contents; and the content adaptation engine, that coordinates the process, selecting the adequate filters based on the data received from the client application.

The top layer of the platform hosts a set of connectors for multiple client applications, namely a web connector, a mobile connector, a CVE connector and an application programming interface.

In the following section we present a use case of a cooperative application that illustrates the usage of the proposed platform.

\section{Use Case: a project management system}

To test the proposed platform, a project management system is also being specified. This system aims to enable the management of all activities related with project management and uses the new SAGA platform to provide the set of services that are required for this purpose.

The project management system requires the following features:

- Authentication.

- Group management.

- Group calendar.

- Document Sharing.

- Shared whiteboard.

- User's awareness

- Communication: instant messaging and videoconferencing.

- Workflow management.

- Group editing.

For this purpose, the project management system uses the two composed services mentioned before, awareness and brainstorming, as well as some of the atomic cooperative services, such as authentication and the group editor. In the latter case, apart from its inclusion in the brainstorming service, group editing can also be used in more restrict situations (e.g., anecdotic synchronous cooperation between two users or asynchronous cooperation). The same occurs for the whiteboard, instant messaging, calendar and file sharing services. 
Some features require the composition of specific services, namely Group calendar and Videoconferencing. The Group calendar service is a composition of the calendar service with group management service, in order to enable the constitution of a specific calendar that can only be accessed and updated by the persons involved in the project being managed. For this purpose, the group calendar also depends on the context service. Regarding the communication features, the instant messaging system relies mainly on the instant messaging service provided by SAGA/R, but videoconferencing requires a combination of external videoconferencing services or applications and the group management service.

Fig. 4 gives a vision of a typical scenario of using the project management system, showing the services that are used in several situations that occur throughout the project lifetime.

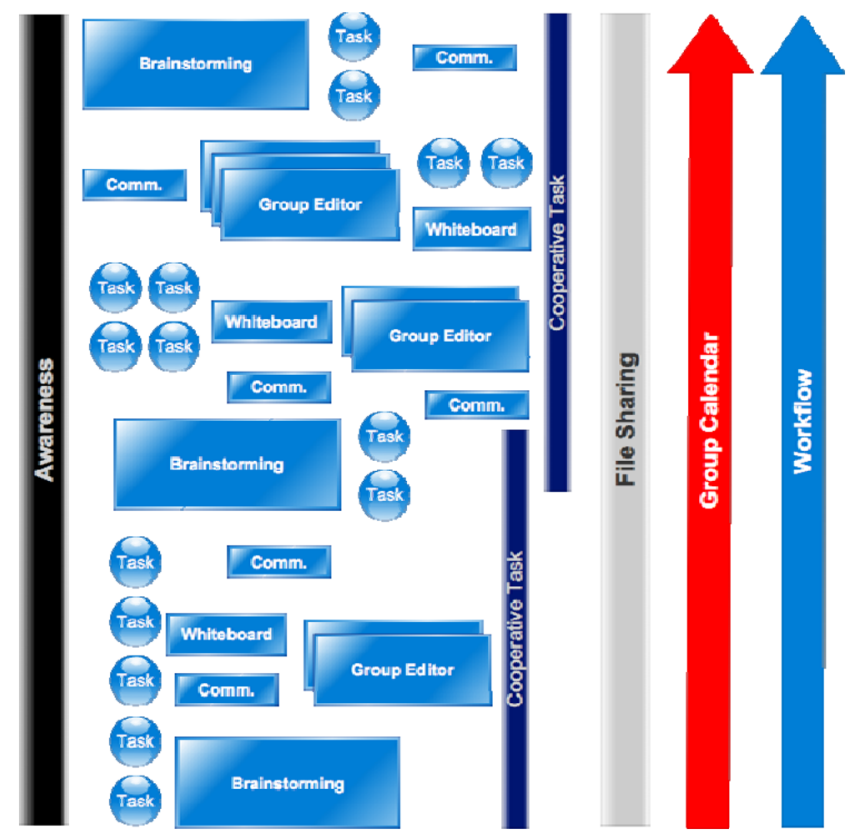

Fig. 4 - Typical usage of the project management system

\section{Final Remarks}

SAGA has been developed in the past to enable the development of cooperative applications based on a set of core web services. The evolution of several related technologies showed new insights and ways to evolve the platform, through the inclusion of new features, such as the regulation of social interaction, the availability of additional communication technologies, the composition of services and the connection to external services and applications. It was also important to reorganize the set of available services, now called atomic services, dividing them into system services and basic cooperative services, while providing ways to combine them to form other composite services, with more complex functionalities. Moreover, composite services that are common to many cooperative applications were also specified, namely brainstorming and awareness services.

Finally, a use case was presented, more precisely a project management system. This system uses several of the new SAGA/R services to build a set of cooperative tools to cope with crucial activities of a project's design and execution.

\section{References}

[1] M.S. Ackerman and E. Mandel, "Memory in the Small: Combining Collective Memory and Task Support for a Scientific Community," Journal of Organizational Computing and Electronic Commerce, Vol. 9, p. 105., vol. 9,1999 , p. 105.

[2] G. Bao-Qing, F. Xiu-Fen, and X. Su-Xia, "P2P Distributed Cooperative Work Model Based on JXTA Platform," Advanced Parallel Processing Technologies, 2007, pp. 658-665; http://dx.doi.org/10.1007/978-3-54076837-1_71.

[3] J. Brandenburg et al., "Artefact: a framework for lowoverhead Web-based collaborative systems," Proceedings of the 1998 ACM conference on Computer supported cooperative work, Seattle, Washington, United States: ACM, 1998, pp. 189-196; http://portal.acm.org/citation.cfm?id=289444.289493.

[4] A. Chabert et al., "Java object-sharing in Habanero," Commun. ACM, vol. 41, 1998, pp. 69-76.

[5] Chen Hua, Qian Jianfei, and He Qingming, "A P2P Architecture for Supporting Group Communication in CSCW Systems," Computer Supported Cooperative Work in Design, 2006. CSCWD '06. 10th International Conference on, 2006, pp. 1-5.

[6] I. Clarke et al., "Freenet: a distributed anonymous information storage and retrieval system," International workshop on Designing privacy enhancing technologies: design issues in anonymity and unobservability, Berkeley, California, United States: Springer-Verlag New York, Inc., 2001, pp. 46-66; http://portal.acm.org/citation.cfm?id=371977\&dl=GUIDE $\&$ coll $=$ GUIDE $\&$ CFID $=10747584 \&$ CFTOKEN $=4055033$ 7.

[7] G. Cugola and G.P. Picco, "Peer-to-Peer for Collaborative Applications," Proceedings of the 22nd International Conference on Distributed Computing Systems, IEEE Computer Society, 2002, pp. 359-364; http://portal.acm.org/citation.cfm?id=646854.708212.

[8] A.K. Dey, S. Daniel, and G.D. Abowd , "Conceptual Framework and a Toolkit for Supporting the Rapid Prototyping of Context-Aware Applications," HumanComputer Interaction (HCl) Journal, vol. 16, 2001, pp. 97166.

[9] M. Dignum et al., "An Organization-oriented Model for Agent Societies," 2002.

[10] C. Ferraris and C. Martel, "Regulation in groupware: the example of a collaborative drawing tool for young children," Groupware, 2000. CRIWG 2000. Proceedings. Sixth International Workshop on, 2000, pp. 119-127.

[11] B. Fonseca and E. Carrapatoso, "SAGA: A Web Services Architecture for Groupware Applications," Groupware: Design, Implementation, and Use, 2006, pp. 246-261; http://dx.doi.org/10.1007/11853862_20.

[12] T. Gross and W. Prinz, "Modelling Shared Contexts in Cooperative Environments: Concept, Implementation, and Evaluation," Comput. Supported Coop. Work, vol. 13, 2004, pp. 283-303.

[13] J. Grudin, "Why CSCW applications fail: problems in the design and evaluationof organizational interfaces," Proceedings of the 1988 ACM conference on Computersupported cooperative work, Portland, Oregon, United States: ACM, 1988, pp. 85-93; http://portal.acm.org/citation.cfm?id=62273. 
[14] T. Gu, H.K. Pung, and D.Q. Zhang, "A service-oriented middleware for building context-aware services," J. Netw. Comput. Appl., vol. 28, 2005, pp. 1-18.

[15] A. Guicking, P. Tandler, and P. Avgeriou, "Agilo: A Highly Flexible Groupware Framework," Groupware: Design, Implementation, and Use, 2005, pp. 49-56; http://dx.doi.org/10.1007/11560296_4.

[16] Hua Xia et al., "PCSCW: P2P-based computer supported cooperative work research," Intelligent Control and Automation, 2002. Proceedings of the 4th World Congress on, 2002, pp. 2770-2774 vol.4.

[17]Q. Jones et al., "People-to-People-to-Geographical-Places: The P3 Framework for Location-Based Community Systems," Comput. Supported Coop. Work, vol. 13, 2004, pp. 249-282.

[18] T. Kindberg, "Mushroom: A framework for collaboration and interaction across the Internet," Proc. CSCW \& the Web, 5th ERCIM workshop, Busbach, 1996, pp. 43--53.

[19] J.H. Lee et al., "Supporting multi-user, multi-applet workspaces in CBE," Proceedings of the 1996 ACM conference on Computer supported cooperative work, Boston, Massachusetts, United States: ACM, 1996, pp. 344-353; http://portal.acm.org/citation.cfm?id=240080.240326.

[20] D. Li and R.R. Muntz, "A collaboration specification language," Proceedings of the 2nd conference on Conference on Domain-Specific Languages - Volume 2, Austin, Texas: USENIX Association, 1999, pp. 12-12; http://portal.acm.org/citation.cfm?id=1267948.

[21] P. Lopez and A. Skarmeta, "ANTS framework for cooperative work environments," Computer, vol. 36, 2003 , pp. 56-62.

[22] N. Luo et al., "Towards Context-Aware Composition of Web Services," Proceedings of the Fifth International Conference on Grid and Cooperative Computing, IEEE Computer Society, 2006, pp. 494-499; http://portal.acm.org/citation.cfm?id=1170137.1170638\& coll=GUIDE \&dl=GUIDE.

[23] S. McIlraith, T. Son, and Honglei Zeng, "Semantic Web services," Intelligent Systems, IEEE, vol. 16, 2001, pp. 46-53.

[24] D.D. Mittleman et al., "Toward a Taxonomy of Groupware Technologies ," Groupware: Design, Implementation, and Use, 2008, pp. 307-320.

[25] J. Munson, "Collaboration Bus Infrastructure: Bus Agents"; http://www.cs.unc.edu/ munson/DARPA/busagent.html.

[26] R.B. Neto et al., "A Web Service Approach for Providing Context Information to CSCW Applications," Proceedings of the WebMedia $\backslash \&$ LA-Web 2004 Joint Conference 10th Brazilian Symposium on Multimedia and the Web 2nd Latin American Web Congress, IEEE Computer Society, 2004, pp. 46-53; http://portal.acm.org/citation.cfm?id=1034017.

[27] T. O'Reilly, "What Is Web 2.0: Design Patterns and Business Models for the Next Generation of Software," Mar. 2007; http://mpra.ub.uni-muenchen.de/4578/.

[28] H. Olivier and N. Pinkwart, "Computer Supported Cooperative Work In The Second Life? ", 2007; http://cscw.in.tuclausthal.de/pdf/CSCW\%20in\%20Second\%20Life\%20_ $\% 20$ camera\%20ready.pdf.

[29] P. Orozco et al., "A Decoupled Architecture for ActionOriented Coordination and Awareness Management in CSCL/W Frameworks," Groupware: Design, Implementation and Use, 2004, pp. 246-261; http://www.springerlink.com/content/f0g1 b1 2 hmvhg5lbn

[30] C. Papadopoulos, "Improving Awareness in Mobile CSCW," Mobile Computing, IEEE Transactions on, vol. 5, 2006, pp. 1331-1346.

[31] H. Paredes and F. Martins, "Social Theatres: A WebBased Regulated Social Interaction Environment," Groupware: Design, Implementation, and Use, 2007, pp. 87-94; http://dx.doi.org/10.1007/978-3-540-74812-0_7.

[32] N. Preguiça et al., "Integrating Synchronous and Asynchronous Interactions in Groupware Applications," Groupware: Design, Implementation, and Use, 2005, pp. 89-104; http://dx.doi.org/10.1007/11560296_7.

[33] K. Salmenjoki and T. Welzer, "Using Web Services and Semantic Web for Producing Intelligent Context-Aware Services," Knowledge-Based Intelligent Information and Engineering Systems, 2004, pp. 1032-1038; http://www.springerlink.com/content/tk226qqp0tx3fp40.

[34] B. Schilit, N. Adams, and R. Want "Context-aware computing applications," Mobile Computing Systems and Applications, 1994. Proceedings., Workshop on, 1994, pp. 85-90.

[35] J.P. Sousa, Eurico Carrapatoso, and Benjamim Fonseca, "Uma Arquitectura para a composição dinâmica de servicos dependentes do contexto "Actas da conferência IADIS Ibero-Americana, UTAD, Vila Real, Portugal: 2007.

[36] J. Trevor, T. Koch, and G. Woetzel, "MetaWeb: bringing synchronous groupware to the world wide web," Proceedings of the fifth conference on European Conference on Computer-Supported Cooperative Work, Lancaster, UK: Kluwer Academic Publishers, 1997, pp. 65-80; http://portal.acm.org/citation.cfm?id=1241985.

[37] "Second Life," Virtual worlds, avatars, 3D chat, online meetings - Second Life; http://secondlife.com/.

[38] "Active Worlds," Home of the 3D Chat, Virtual Worlds Building Platform; http://www.activeworlds.com/. 